\title{
Evaluación de la resistencia mecánica de la unión soldada en la aleación de aluminio 6261
}

\author{
Maribel Amu-Bolaños*, Fernando Franco-Arenas* $\$$ \\ * Escuela de Ingeniería de Materiales, Universidad del Valle. Cali - Colombia \\ ${ }^{\S}$ e-mail: ffrancoa@univalle.edu.co
}

(Recibido: Octubre 5 de 2005 - Aceptado: Enero 18 de 2006)

\begin{abstract}
Resumen
Se estudia la relación entre la temperatura pico, la microdureza, la microestructura, la ubicación de la falla y la resistencia en la zona térmicamente afectada de juntas soldadas por el proceso GMAW en aleación de aluminio 6261-T6 y T5. Se logró una buena correlación de los resultados con la distribución de los precipitados reportados en la literatura. Los perfiles de temperatura pico se compararon con los perfiles de microdureza. Se encontró un punto de mínima microdureza en la ZAC con un valor menor del $70 \%$ del metal base, donde también se presentó la falla en tensión, correspondiendo a una temperatura de $380^{\circ} \mathrm{C}$.
\end{abstract}

Palabras Clave: Aleaciones de aluminio, Soldadura por arco GMAW, Zona afectada por el calor, Endurecimiento por envejecimiento, Microdureza y microestructura.

\begin{abstract}
The relationship between peak temperatures, microhardness, microstructure, failure location and strength in the heat-affected zone (HAZ) of gas metal arc (GMAW) welded joints of extruded aluminum alloy 6261-T6 and T5 were investigated. The experimental data obtained have a good correlation with precipitate distribution reported in the literature. This resulted in a plane of minimum hardness within the HAZ where hardness may be less than $70 \%$ of that for the base metal. Failure during tensile testing typically occurred through this region regardless of the welding variables used. The peak temperature profiles were determined in the HAZ and compared with the corresponding hardness profiles. It was found that the minimum hardness and the location of failure of the tensile specimens in the $\mathrm{HAZ}$ occurred in the region subjected to a temperature of $716^{\circ} \mathrm{F}\left(380^{\circ} \mathrm{C}\right)$.
\end{abstract}

Key Words: Aluminum alloys, Gas metal arc welding, Heat affected zone, Age hardening, Microhardness and microstructure.

\section{Introducción}

El uso de aleaciones de aluminio de alta resistencia en la fabricación de estructuras soldadas y vehículos de transporte se ha incrementado debido a la existencia de aleaciones soldables con una elevada resistencia específica y excelente resistencia a la corrosión comparadas con los aceros de construcción.
El proceso de endurecimiento principal de las aleaciones de aluminio serie 6XXX se logra con el tratamiento térmico de envejecimiento que se desarrolla en tres pasos, ver figura 1. Inicialmente, la aleación es llevada a una temperatura en el rango de existencia de la solución sólida á $\left(30^{\circ} \mathrm{C}\right.$ aprox. por debajo de la temperatura eutéctica); posteriormente, 
la aleación es templada rápidamente hasta temperatura ambiente para retener la solución sólida sobresaturada. En el último paso, la aleación es calentada a la temperatura de envejecimiento con el propósito de lograr un precipitado coherente fino y disperso en la matriz de una solución sólida rica en aluminio.
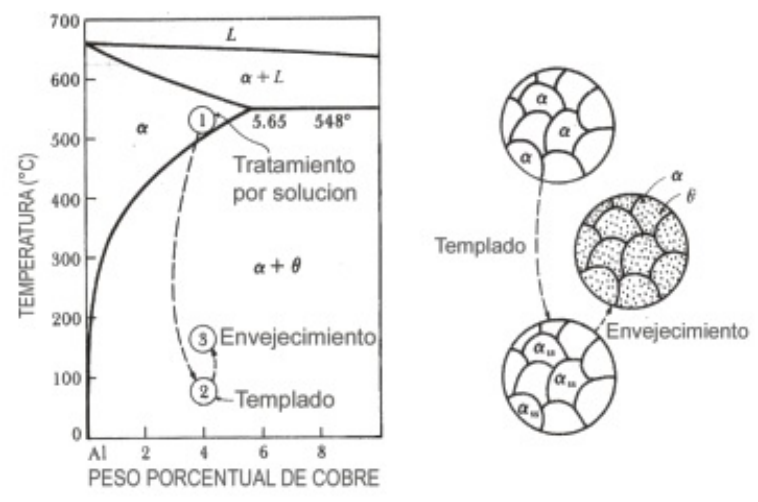

Figura 1. Etapas del envejecimiento

Para el sistema de aleación Al-Mg-Si con contenidos de $0.61 .2 \%$ de $\mathrm{Mg}$ y $0.41 .3 \%$ de $\mathrm{Si}$; la secuencia de formación de los precipitados es como sigue:

$$
\mathrm{SS} \rightarrow \mathrm{GP} \rightarrow \beta^{\prime \prime}\left(\mathrm{Mg}_{2} \mathrm{Si}\right) \rightarrow \beta^{\prime}\left(\mathrm{Mg}_{2} \mathrm{Si}\right) \rightarrow \beta\left(\mathrm{Mg}_{2} \mathrm{Si}\right)
$$

Donde SS denota la solución sólida sobresaturada; GP, $\beta^{\prime \prime}, \beta^{\prime}$ y $\beta$ los productos de la reacción de precipitación. Las zonas de Guinnier-Preston (GP) son agujas orientadas en la dirección 001 coherentes con la estructura cristalina de la solución sólida, con $\beta^{\prime}$ (semicoherente) y $\beta^{\prime \prime \prime}$ (coherente) mostrando una orientación similar. Messler ${ }^{(1)}, \mathrm{Kou}^{(2)}$.

Para $\operatorname{Malin}^{(3)}$ la máxima resistencia de la aleación 6061 ocurre cuando se envejece para producir una estructura de agujas $\left(\beta^{\prime \prime}\right)$ sobre una matriz de á. Mientras que la contribución de la fase $\beta^{\prime}$ es moderada y las otras partículas $\beta$ afectan muy poco la resistencia.

Durante el proceso de soldadura GMAW de aleaciones de aluminio de la serie 6XXX, el calor aportado afecta el material dando origen a cambios termodinámicos que modifican las propiedades mecánicas y metalúrgicas de la unión. Se pueden reconocer dos regiones bien marcadas: la zona de fusión (ZF) y la zona afectada por el calor (ZAC). (ver figura 2). La microestructura final de estas zonas de la unión soldada está principalmente determinada por la velocidad de enfriamiento desde la temperatura pico alcanzada en cada punto durante el ciclo térmico de la soldadura.

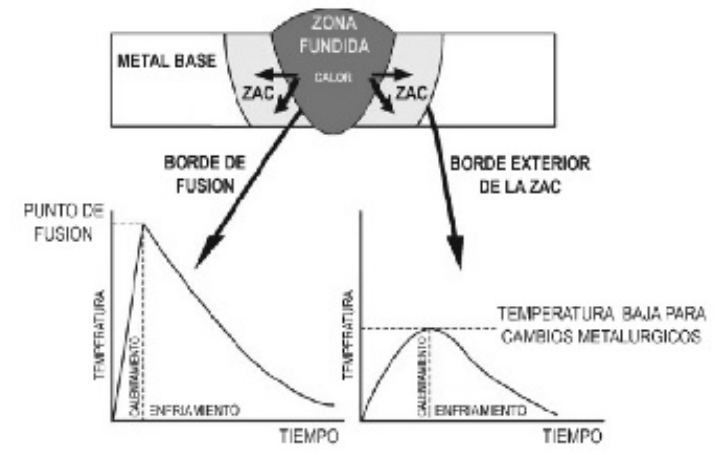

Figura 2. Ciclo térmico de la soldadura

La zona parcialmente fundida (ZPF) alcanza temperaturas pico entre la temperatura de liquidus y la temperatura eutectica de la aleación, que disuelve los precipitados y los principales elementos de aleación presentes en los bordes de grano adyacentes a la línea de fusión. En esta zona se puede presentar usualmente la licuación de los bordes de grano y hasta fisuras en caliente por efecto de los esfuerzos internos de la soldadura.

La zona afectada por el calor (ZAC) alcanza temperaturas altas, por debajo de la temperatura de liquidus de la aleación, las cuales generan diferentes cambios microestructurales sobre los precipitados endurecedores dependiendo de la temperatura pico lograda en cada punto. La ZAC puede subdividirse en varias regiones:

La zona de solución alcanza una temperatura máxima entre la temperatura eutéctica y la temperatura de solvus de la aleación. Los precipitados se disuelven durante el calentamiento y luego durante el enfriamiento pueden volver a precipitar en el interior o en los bordes de grano; o la solución sólida puede 
permanecer sobresaturada en soluto, dependiendo de la velocidad de enfriamiento producida.

La zona siguiente alcanza temperaturas pico entre la temperatura de solvus y la temperatura de envejecimiento del material base. Se caracteriza por el crecimiento de los precipitados metaestables y la transformación en fases más estables de acuerdo a la secuencia de precipitación de la aleación. En esta zona la resistencia mecánica es reducida y se detecta una disminución local de la dureza debida a la reversión del precipitado endurecedor $\beta^{\prime \prime}$ en $\beta^{\prime}$ e incluso en $\beta$.

Si las juntas soldadas de aleaciones 6261T6 se someten a tratamientos térmicos de envejecimiento posteriores a la soldadura, se espera que ocurran cambios microestructurales en la ZAC y la ZF que produzca una recuperación total o parcial de las propiedades mecánicas perdidas durante la soldadura.

Lawson $^{(4)}$ obtuvo hasta un $100 \%$ de recuperación de las propiedades mecánicas de la unión soldada de aleaciones de aluminio 6061, respecto del metal base con un tratamiento total; mientras que obtuvo hasta un $70 \%$ de recuperación con un tratamiento parcial de sólo envejecimiento. Por otra parte Guiterrez ${ }^{(5)}$ evaluó los cambios de dureza y resistencia mecánica en uniones soldadas de aleaciones de aluminio 6063 T6.

\section{Procedimiento experimental}

En el presente artículo se estudió el cambio de las propiedades mecánicas de uniones soldadas de láminas de aleación de aluminio tipo 6261 en condición de tratamiento térmico T5 y T6 de $3 \mathrm{~mm}$ de espesor de producción nacional. Estas láminas se usan para la construcción de formaletas o páneles que conforman el molde para el vaciado del concreto en la construcción de casas y apartamentos de vivienda.

El material de aporte usado fue la aleación de aluminio ER-5356 con diámetro de $1.2 \mathrm{~mm}$. La tabla 1 muestra la composición química de los materiales usados. Se realizaron uniones de penetración total de una sola pasada, a tope cuadrado sin bisel, con el proceso de soldadura GMAW usando corriente DCEP y utilizando argón como gas de protección $(30 \mathrm{CFH})$. La tabla 2 muestra las condiciones de soldadura usadas para cada probeta y definidas a partir de $\mathrm{ASM}^{(6)}$ y The Aluminum Assoc. (7)

Tabla 1. Composición química de los materiales base y de aporte.

\begin{tabular}{ccccccccccc}
\hline & & \multicolumn{1}{c}{ COMPOSICIÓN QUÍMICA, \% } & & \\
$\begin{array}{c}\text { ALEACIONES } \\
\text { DE ALUMINIO }\end{array}$ & $\mathbf{S i}$ & $\mathbf{C u}$ & $\mathbf{M n}$ & $\mathbf{M g}$ & $\mathbf{C r}$ & $\mathbf{Z n}$ & $\mathbf{T i}$ & $\mathbf{F e}$ & $\mathbf{A l}$ \\
\hline AA 6261 & 0,56 & 0,18 & 0,25 & $\mathbf{0 . 8 5}$ & 0,01 & 0,036 & 0,02 & 0,38 & rem. \\
ER 5356 & 0,25 & 0,10 & 0,12 & 5,00 & 0,12 & 0,10 & 0,13 & 0,40 & rem. \\
\hline
\end{tabular}

Las probetas se prepararon según las recomendaciones del código estructural AWS D $1.2^{\left({ }^{(8)}\right.}$ para la calificación de soldadores y procedimiento de soldadura de aleaciones de aluminio. Las pruebas de tensión se realizaron según la especificación ASTM E8. El ensayo de microdureza se realizó mediante un barrido a través de las distintas zonas de la unión soldada, utilizando un microdurómetro Vickers mediante la aplicación de una carga de 300 gramos durante 15 segundos.

Las micrografías fueron tomadas utilizando un microscopio óptico marca Olimpus BX 60 después de atacar las probetas con el reactivo de Keller $(1 \mathrm{ml}$ $\mathrm{HF}, 1.5 \mathrm{ml} \mathrm{HCl}, 2.5 \mathrm{ml} \mathrm{HNO}_{3}$, y $95 \mathrm{ml} \mathrm{H}_{2} \mathrm{O}$ ) durante 30 segundos.

Se evaluó la influencia de los tratamientos térmicos post-soldadura total y parcial sobre las propiedades finales de la unión soldada. Como se muestra en la figura 3, el tratamiento total involucra el tratamiento de solución, temple y envejecimiento. El tratamiento parcial sólo involucra la etapa final de envejecimiento. Se procuraba recuperar la dureza y la resistencia a la tensión perdidas en la ZAC de las uniones soldadas.

Tabla 2. Variables de Operación

\begin{tabular}{cccccc}
$\begin{array}{l}\text { MATERIAL } \\
\text { BASE }\end{array}$ & CONDICIÓN & $\begin{array}{c}\text { CORRIENTE } \\
\text { A. }\end{array}$ & $\begin{array}{c}\text { VOLTAJE } \\
\text { V. }\end{array}$ & $\begin{array}{c}\text { VELOCIDAD } \\
\text { DE } \\
\text { SOLDADURA } \\
\text { mm/s. }\end{array}$ & $\begin{array}{c}\text { CALOR } \\
\text { APORTADO } \\
\text { J/mm. }\end{array}$ \\
\hline \multirow{2}{*}{ AA 6261- T6 } & C1 & 130 & 18.2 & 14.2 & 166.7 \\
& C2 & 134 & 18.0 & 8.8 & 274.7 \\
AA 6261- T5 & C1 & 136 & 18.2 & 13.5 & 183.3 \\
& C2 & 139 & 18.0 & 9.3 & 270.2 \\
\hline
\end{tabular}




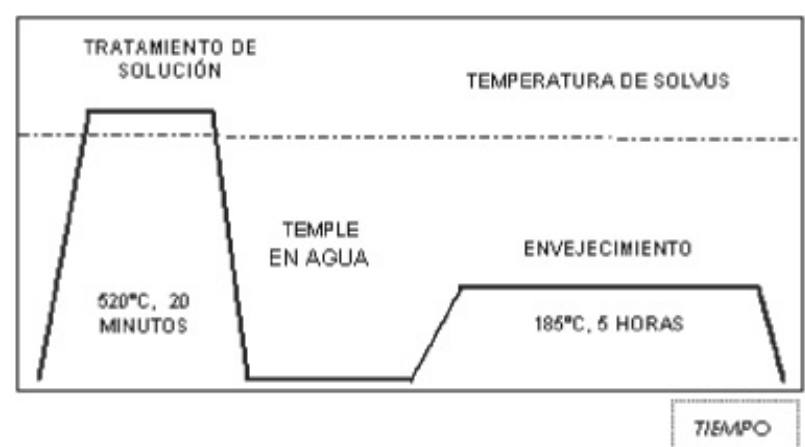

Figura 3. Esquema del tratamiento térmico total post-soldadura

\section{Resultados}

Los perfiles de microdureza obtenidos a nivel experimental y las temperaturas pico calculadas en cada punto (ver figura 4) permiten identificar tres zonas en la unión soldada, tomando como punto de referencia la línea de fusión a en todos los casos. Estas zonas se definen como:

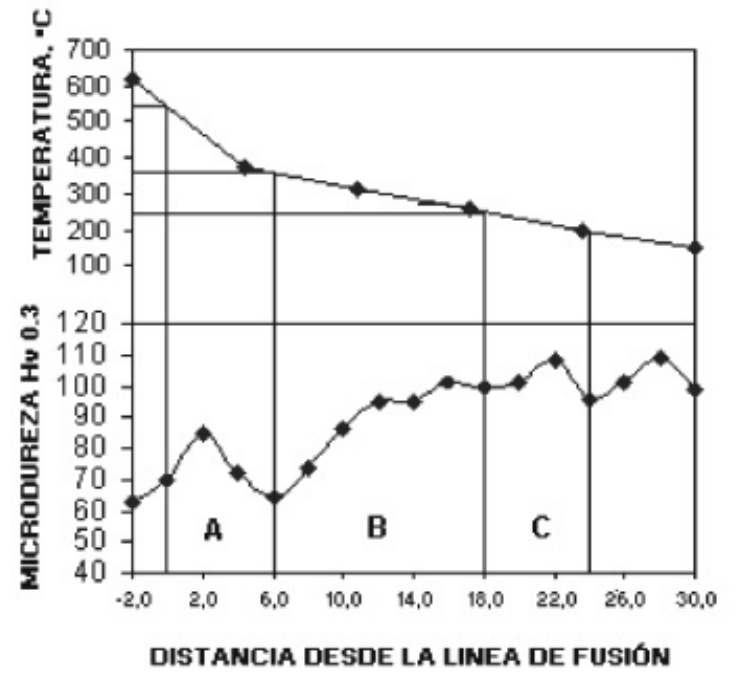

$\longrightarrow-6261$ T6

Figura 4. Definición de las zonas dentro de la ZAC

Zona A: Se encuentra en un rango de temperaturas pico entre $540^{\circ} \mathrm{C}-380^{\circ} \mathrm{C}$. Inicia en la línea de fusión y se extiende hasta el punto de mínima dureza. Cerca a $\operatorname{los} 540^{\circ} \mathrm{C}$ los precipitados endurecedores se disuelven durante el calentamiento y luego en el enfriamiento la microestructura resultante puede ser una solución sólida sobresaturada, o puede estar compuesta por granos de á rodeados de una fase eutéctica de bajo punto de fusión. La resistencia mecánica y la dureza alcanzadas en esta zona después del enfriamiento depende de cualquier precipitación que pueda ocurrir.

El límite de formación del precipitado endurecedor $\beta^{\prime}\left(\mathrm{Mg}_{2} \mathrm{Si}\right)$ es $380^{\circ} \mathrm{C}$; Torres ${ }^{(9)}$, por lo tanto, en esta zona la pérdida de coherencia es total (sobreenvejecimiento) por la transformación del precipitado metaestable y semicoherente $\beta^{\prime}\left(\mathrm{Mg}_{2} \mathrm{Si}\right)$ en el precipitado $\beta\left(\mathrm{Mg}_{2} \mathrm{Si}\right)$ no coherente de equilibrio. Para una unión soldada en particular, este cambio microestructural es reflejado por un punto de mínima dureza en la interfase entre la zona A y la Zona B.

Zona B: Corresponde a un rango de temperaturas entre $380^{\circ} \mathrm{C}-240^{\circ} \mathrm{C}$, donde el precipitado endurecedor $\beta^{\prime}\left(\mathrm{Mg}_{2} \mathrm{Si}\right)$ crece y pierde coherencia con la matriz. Este comportamiento afecta notoriamente la dureza y resistencia mecánica dentro de esta zona. La pérdida de coherencia por crecimiento del precipitado disminuye con el aumento de la distancia desde la línea de fusión.

Zona C: Corresponde con el intervalo de temperaturas de $240^{\circ} \mathrm{C} \quad 160^{\circ} \mathrm{C}$. El límite inferior corresponde a la temperatura de formación de la fase $\beta^{\prime \prime}\left(\mathrm{Mg}_{2} \mathrm{Si}\right)$, la cual existe a temperaturas inferiores a $240^{\circ} \mathrm{C}$. En los perfiles de microdureza la transformación del precipitado $\beta^{\prime \prime}\left(\mathrm{Mg}_{2} \mathrm{Si}\right)$ es detectado por una disminución local de la dureza. En esta zona la microdureza presenta fluctuaciones causadas por la presencia de puntos suaves locales que no tienen un efecto significativo en la resistencia de la unión. En ninguno de los casos experimentales la falla ocurre en esta zona.

\section{Mediciones de Microdureza.}

Las figuras 5 y 6 muestran el efecto de las dos condiciones de soldadura $\mathrm{C} 1$ y $\mathrm{C} 2$ sobre las microdurezas en la unión soldada de la AA 6261 con tratamiento térmico inicial T6 y T5, respectivamente. Se puede también evaluar el efecto del calor aportado, asociado a cada condición C1 y C2, sobre el 
grado de cambio metalúrgico.

Los perfiles de microdureza mantienen la misma forma independiente de los parámetros de soldadura usados; sin embargo, se aprecia especialmente en las zonas A y B, que a mayor aporte de calor (condición C2) se presentaron menores valores de dureza, lo cual corresponde a un mayor grado de sobreenvejecimiento por efecto de la soldadura.

Para el caso de la AA 6261-T6, ver figura 5, un aumento de calor del $39 \%$, produce una reducción del $28 \%$ en la microdureza y del $14 \%$ en la resistencia a la tensión, con respecto a las propiedades iniciales del material base.

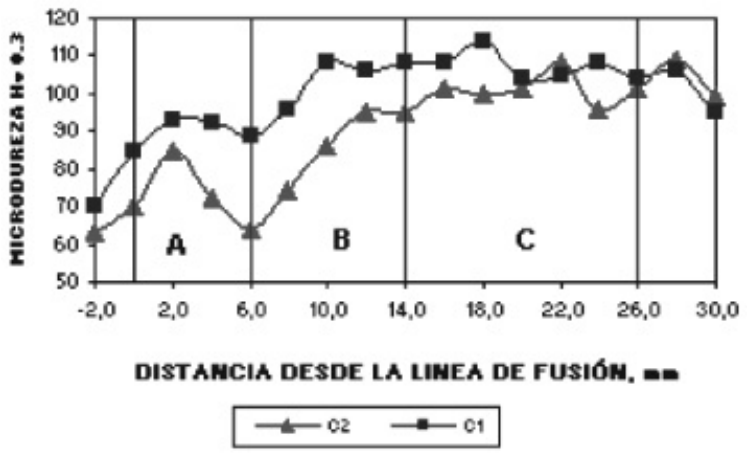

Figura 5. Perfiles de microdureza para la AA6261-T6

Para la AA6261-T5, ver figura 6, un aumento de calor del $32 \%$ produce una reducción de $24 \%$ en la microdureza y de $12 \%$ en la resistencia a la tensión.

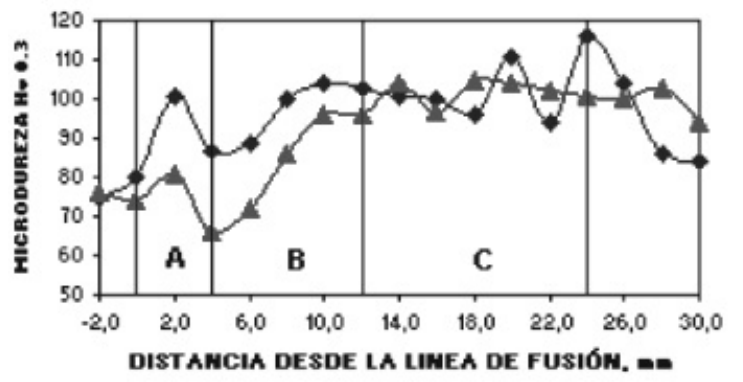

$$
\longrightarrow-\mathrm{Or}^{\longrightarrow} \longrightarrow \mathrm{er}_{2}
$$

Figura 6. Perfiles de microdureza para la AA6261-T5

\section{Análisis Metalográfico}

En las figuras 7, se muestran las microestructuras típicas encontradas en cada una de las zonas de la unión soldada. La zona fundida (figura 7A),

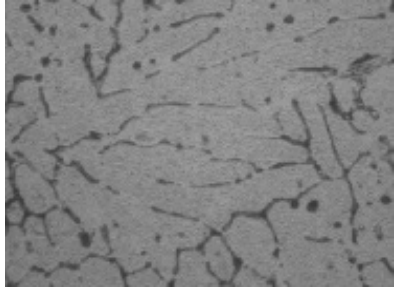

7a. Zona fundida. $500 x$

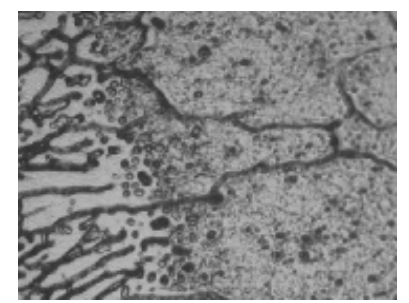

7b. Zona parcialmente fundida. 200x.

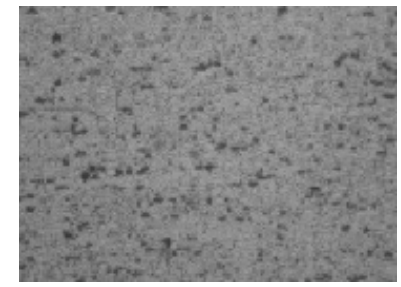

7c. Metal base no afectado. 200x.

Figura 7. Microestructuras en la Unión Soldada

compuesta mayoritariamente por el aporte ER-5356, muestra una microestructura típica de fundición que inicia con un crecimiento epitaxial de los granos columnares dendríticos desde la línea de fusión pero que cambian a granos equiaxiales hacia el interior de la zona fundida.

En el límite de la ZF se aprecian gruesas líneas de licuación que se proyectan hacia la ZAC y que marcan la presencia de la zona parcialmente fundida. Ver figura $7 \mathrm{~B}$.

La zona afectada por el calor, donde el calor aportado por el proceso de soldadura afecta a los precipitados 
endurecedores que no son estables termodinámicamente, no se alcanza a resolver los detalles microestructurales con el microscopio óptico.

El metal base no afectado, correspondiente a las zonas donde las temperaturas alcanzadas no fueron lo suficientemente altas para producir cambios microestructurales notables, se puede ver en la figura 7C.

\section{Ensayos de Tensión}

Durante el ensayo de tensión, las uniones soldadas fallaron bajo un comportamiento dúctil pero con poca deformación plástica. La microdureza mínima y la resistencia mecánica mantienen una relación directa; al disminuir la microdureza mínima, disminuye también la resistencia a la tensión para una misma condición o procedimiento de soldadura. Esta relación también está afectada por el calor aportado durante el proceso de soldadura. Al aumentar el calor aportado disminuyen la dureza mínima y la resistencia a la tensión obtenidas.

La tabla 3 muestra los resultados del efecto de dos niveles de aporte de calor sobre la resistencia a la tensión en las aleaciones AA6261 T6y T5.

Tabla 3. Resultados de los ensayos de tensión.

\begin{tabular}{|c|c|c|c|c|c|c|c|}
\hline ALEACIÓN & CONDICIÓN & $\underset{\mathrm{Kg} / \mathrm{mm}^{2}}{\sigma_{\mathrm{t}}}$ & $\underset{\mathrm{Kg} / \mathrm{mm} \mathrm{m}^{2}}{\sigma_{\mathrm{y}}}$ & \% ELONG. & \% REDUCCIÓN & $\begin{array}{l}\text { DUREZA } \\
\text { MiN. } \\
\text { Hv } 0.3\end{array}$ & $\begin{array}{c}\text { CALOR } \\
\text { APORTE } \\
\text { J/mm. }\end{array}$ \\
\hline \multirow{2}{*}{ AA $6261 \mathrm{~T} 6$} & $\mathrm{Cl}$ & 23.70 & 22.90 & 8.76 & 10.75 & 89 & 166.0 \\
\hline & $\mathrm{C} 2$ & 20.23 & 19.65 & 9.60 & 23.74 & 64 & 274.7 \\
\hline \multirow{2}{*}{ AA $6261 \mathrm{~T} 5$} & $\mathrm{Cl}$ & 22.60 & 22.26 & 5.36 & 12.82 & 89 & 183.2 \\
\hline & $\mathrm{C} 2$ & 19.80 & 19.42 & 8.20 & 23.61 & 66 & 270.2 \\
\hline
\end{tabular}

\section{Tratamiento térmico post-soldadura}

En las figuras 8 y 9 se muestra el efecto de los tratamientos térmicos post-soldadura total y parcial sobre los perfiles de dureza para AA 6261-T6 y T5, respectivamente; mientras que en las figuras 10 y 11 se muestran los cambios microestructurales en las distintas zonas de la unión soldada resultantes de los tratamientos térmicos postsoldadura parcial y total.

El tratamiento térmico parcial, es decir, sólo la etapa de envejecimiento de la junta soldada, produjo un notable incremento de la dureza en las regiones Ay B de la unión soldada. Donde la microestructura constaba de una solución sólida sobresaturada, precipito $(\mathrm{Mg} 2 \mathrm{Si})$ y/o $(\mathrm{Mg} 2 \mathrm{Si})$ por efecto del tratamiento térmico parcial. Por su parte, el precipitado (Mg2Si), coherente y estable, no se modificó; esto hace, que se mantenga un punto de mínima dureza en el límite de existencia entre la zona A y la zona B.

El metal base sin afectar muestra muy poca respuesta al tratamiento parcial debido a que éste corresponde básicamente al envejecido inicial del metal base antes de soldar.

El tratamiento térmico total produce una recuperación completa de la dureza en el interior de la ZAC con respecto a los valores reportados para el metal base inicial. En la ZAC se espera lograr homogeneidad de las propiedades mecánicas después del tratamiento térmico completo. Los precipitados deben disolverse, formar una solución sólida y mantenerla al finalizar la etapa de temple; durante el envejecimiento deben precipitar nuevas fases endurecedoras de acuerdo a lo establecido por la secuencia de precipitación característica de esta aleación.

En el caso de la aleación soldada en condición de T6, ver figura 8 , el tratamiento térmico completo produce una recuperación de dureza de $46 \%$ en el punto de mínima dureza (6 mm de la línea de fusión) y de 19 $\%$ en el límite de existencia de la zona B $(16 \mathrm{~mm}$ de la línea de fusión).
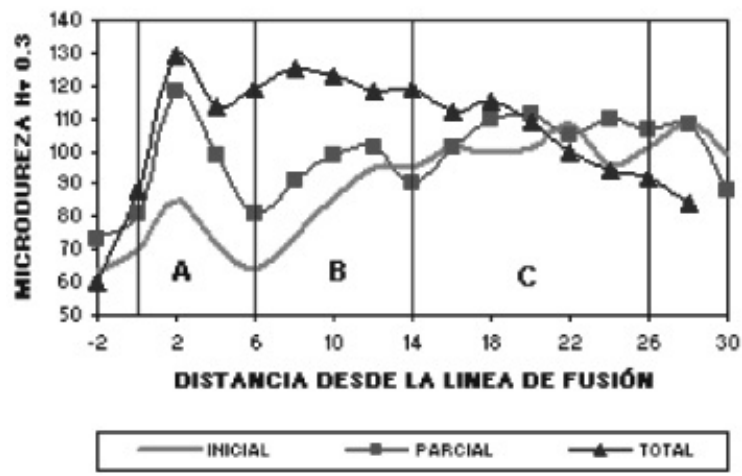

Figura 8. Efecto de los tratamientos térmicos total y parcial para AA 6261 - T6

Para la condición de tratamiento T5, figura 9, el tratamiento térmico completo reporta recuperación de dureza de $49 \%$ en el punto de mínima dureza (4 mm de la línea de fusión) y de $25 \%$ en el limite de existencia de la zona B (12 mm de la línea de fusión). 
Para el tratamiento térmico parcial, la recuperación de microdureza en el punto de mínima dureza es de 21\%; en el límite de existencia de la zona B la recuperación es $4 \%$.

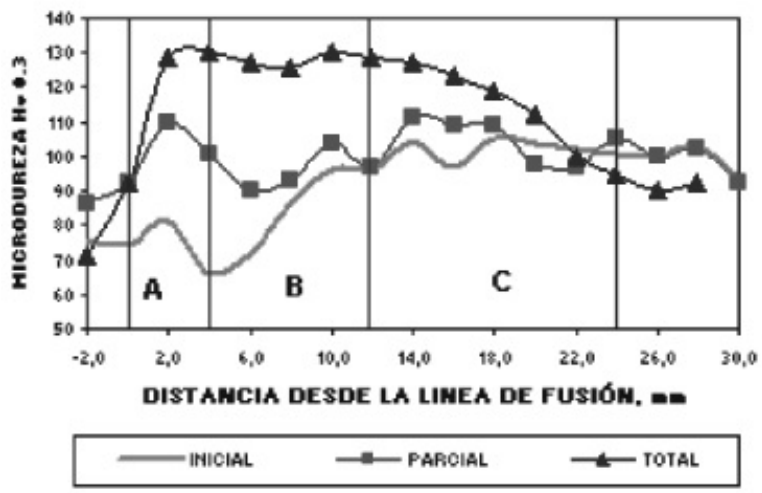

Figura 9. Efecto de los tratamientos térmicos total y parcial para AA 6261 T5

El tratamiento parcial no es suficiente para producir cambios microestructurales significativos en las distintas zonas de la unión soldada. (Ver figura 10).

El tratamiento térmico completo modifica la morfología de la ZF pero mantiene la evidencia de la microestructura dendrítica de la zona fundida (figura 11A).

La microestructura de la ZAC es modificada totalmente y las líneas de licuación son eliminadas totalmente de la zona parcialmente fundida (figura 11B). El metal base (figura 11C) muestra partículas extremadamente pequeñas de los precipitados finamente dispersas en una matriz de aluminio.

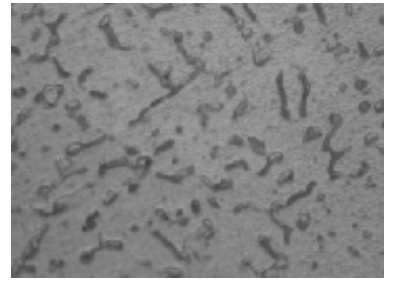

10a. Zona fundida. 500x.

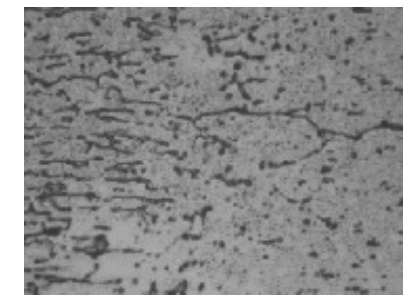

10b. Zona parcialmente fundida. $200 \mathrm{x}$.

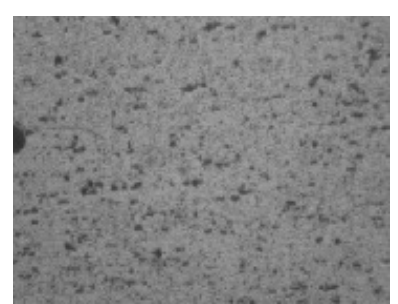

10c. Metal base no afectado. 200x.

Figura 10. Tratamiento térmico parcial

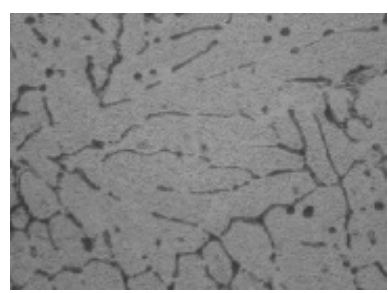

11a. Zona fundida. 500x.

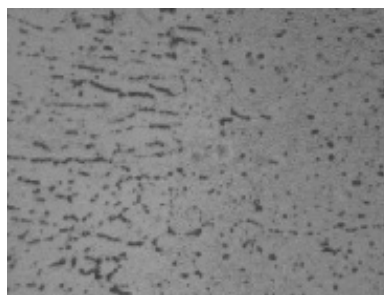

11b. Zona parcialmente fundida. 200x.

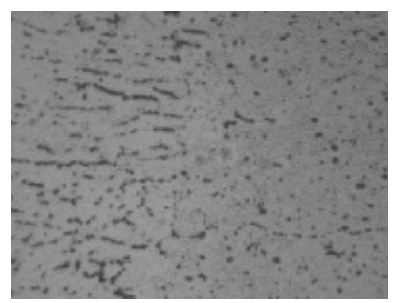

11c. Metal base no afectado. 200x

Figura 11. Tratamiento térmico total

\section{Conclusiones}

El aporte de calor de la soldadura tiene un efecto sensible sobre los cambios microestructurales y de propiedades mecánicas especialmente en la ZAC de las aleaciones de aluminio 6261 , independientemente del tratamiento térmico inicial T5 o T6. 
La caída de resistencia mecánica y dureza más notoria en la ZAC, es el resultado del sobreenvejecimiento producido sobre la AA 6261 envejecida por efecto del ciclo térmico de soldadura.

La microdureza y la resistencia mecánica mantienen una relación directa; al disminuir la microdureza en el punto de falla, disminuye la resistencia a la tensión para una misma condición o procedimiento de soldadura.

El tratamiento térmico de envejecimiento total aplicado posterior a la soldadura, propició una modificación importante en la microestructura de la ZAC y una recuperación de la microdureza a valores cercanos a los del metal base.

El tratamiento térmico de envejecimiento parcial produce una recuperación limitada de microdureza en la ZAC , debido a que el envejecimiento propicia la precipitación de fases endurecedores sólo en aquellas regiones donde existe una solución sólida sobresaturada.

\section{Referencias bibliográficas}

American Welding Society, 1990. Structural Welding Code Aluminum,ANSI/AWSD1.2-90.

ASM Handbook, 1993, Vol. 6, Welding, Brazing and Soldering, Welding of Aluminum Alloys, pag. 722 739.

Guitterez, Neye y Zschech, 1996, Microestructura, perfiles de dureza y resistencia a la tensión en uniones de AA6013 T6. Welding Research Supplement, pag.115-s a 123 -s.

Kou, S. 1987. Welding Metallurgy. John Wiley \& Sons, New York, N.Y.

Lawson, W., Kerr, H.W., 2003, Increasing the strength of GMA Welds in 6000 Series Aluminum Alloys, Canadian Welding Assoc. J., Spring, pp.30 34.

Malin, V., 1995, Study of Metallurgical Phenomena in the HAZ of 6061- T6 Aluminum Welded Joints. Welding Research Supplement, 64, 9, pag. 305-s. a 318-s.
Messler, Jr., R.W., 1999. Principles of Welding. Processes, Physics, Chemistry and metallurgy, John Wiley \& Sons, New York, N.Y.

The Aluminum Association. 2002. Welding Aluminum; Theory and practice. Fourth Edition.

Torres, J., 2002, Evolución microestructural de la aleación de aluminio 6061 durante el proceso de soldadura MIG. Ingeniería \& Desarrollo. Universidad del Norte. No. 12, pag. 52-65. 